\title{
Keeping Plasmodium awake
}

Malaria, which is caused by Plasmodium spp. parasites, remains a great disease burden worldwide. Artemisinin and artemisinin-based combination therapies are the firstline antimalarial drugs; however, resistance to artemisinin has become widespread and, moreover, a small proportion of parasites become latent during treatment and re-emerge (known as recrudescence), resulting in treatment failure. These recrudescent parasite isolates are not drug resistant and the molecular mechanism of artemisinin-induced parasite latency has remained elusive. In this paper, Zhang et al. show that artemisinin-induced latency is mediated by general translational repression following the phosphorylation of the a-subunit of Plasmodium eukaryotic initiation factor 2 (eIF $2 \alpha)$.

Phosphorylation of eIF $2 \alpha$ occurs during cellular stress and leads to decreased protein synthesis to enable cell recovery from stress. Previous reports found that slow-growing and latent stages of apicomplexan parasites, including Plasmodium spp., have increased levels of eIF $2 \alpha$ phosphorylation. On the basis of those findings, the authors hypothesized that phosphorylation of parasite eIF $2 \alpha$ and translational repression contribute to artemisinin-induced latency.

They first determined the phosphorylation status of eIF2 $\alpha$ in malaria parasites treated with derivatives of

artemisinininduced latency is mediated by general translational repression artemisinin as well as in recrudescent parasites after treatment. Mice infected with Plasmodium berghei were treated with artesunate and the authors showed that phosphorylation of parasite eIF $2 \alpha$ was increased following drug treatment, whereas eIF2 $\alpha$ was dephosphorylated in the recrudescent $P$. berghei. Similarly, phosphorylation of eIF $2 \alpha$ in

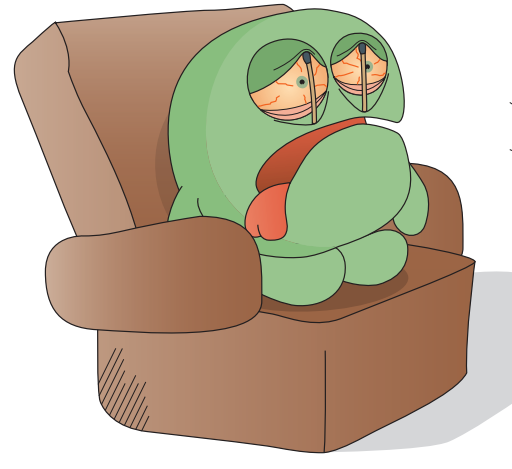

synchronized cultures of Plasmodium falciparum was increased in the presence of dihydroartemisinin. Indeed, high phosphorylation levels of eIF $2 a$ correlated with high rates of recrudescence following drug treatment. Moreover, intensified eIF2 $\alpha$ phosphorylation due to a dephosphorylation inhibitor rendered parasites less sensitive to the antimalarial drugs.

Most clinical antimalarials target blood-stage parasites, which are responsible for the clinical symptoms associated with malaria. The parasite kinase PK4 phosphorylates eIF2 $\alpha$ and is essential for parasite development in erythrocytes. The authors were able to show that exposure to artesunate leads to the oligomerization, autophosphorylation and activation of the ER-resident PK4 and enhanced phosphorylation of eIF $2 \alpha$. In addition, overexpression of a dominant-negative version of PK4 decreased eIF2 $a$ phosphorylation in $P$. berghei, and protein synthesis was increased in these parasites compared with parasites expressing PK4 carrying synonymous mutations (which do not alter the amino acid specificity of the codons). Importantly, no recrudescence after artesunate treatment was observed in parasites transfected with the dominantnegative version of PK4. Moreover, the combination of artemisinin



derivatives and a pharmacological PK4 inhibitor abolished parasite recrudescence in $P$. berghei-infected mice and $P$. falciparum cultures, which suggests that inhibition of PK4 prevents recrudescence and restores the sensitivity of the parasites to the antimalarial drug.

In summary, the results suggest that activated PK4 phosphorylates eIF2 $\alpha$ and inhibits global protein synthesis in the parasites, thus promoting latency and possibly enabling the parasites to recover from the damage accrued during drug exposure. The finding that translational control is crucial for parasite recrudescence might have implications for other pathogenic parasites with latent stages, such as Toxoplasma gondii. As inhibition of PK4 blocked the transition into the latent phase and, consequently, prevented recrudescence following treatment, the authors propose that interference with this stress response pathway may be a viable treatment option to prevent recrudescent infections.

Andrea Du Toit

ORIGINAL ARTICLE Zhang, M. et al. Inhibiting the Plasmodium elF2 $a$ kinase PK4 prevents artemisinin-induced latency. Cell Host Microbe https://doi.org/10.1016/j.chom.2017.11.005 (2017) FURTHER READING Paddon, C. J. \& Keasling, J. D. Semi-synthetic artemisinin: a model for the use of synthetic biology in pharmaceutical development. Nat. Rev. Microbiol. 12, 355-367 (2014) 